\title{
Shape-Based Co-occurrence Matrices for Defect Classification
}

\author{
Rami Rautkorpi and Jukka Iivarinen \\ Helsinki University of Technology, \\ Laboratory of Computer and Information Science, \\ P.O. Box 5400, FIN-02015 HUT, Finland \\ \{rami.rautkorpi, jukka.iivarinen\}@hut.fi
}

\begin{abstract}
This paper discusses two statistical shape descriptors, the Edge Co-occurrence Matrix (ECM) and the Contour Co-occurrence Matrix $(\mathrm{CCM})$, and their use in surface defect classification. Experiments are run on two image databases, one containing metal surface defects and the other paper surface defects. The extraction of Haralick features from the matrices is considered. The descriptors are compared to other shape descriptors from e.g. the MPEG-7 standard. The results show that the ECM and the CCM give superior classification accuracies.
\end{abstract}

\section{Introduction}

Shape features can be divided into two main categories [1]: syntactical and statistical. Syntactical features use structural descriptions that are suitable for regular shapes, such as those of man-made objects, while statistical features are more suitable for irregular, naturally occurring shapes. In surface defect inspection, the defect types can have very similar overall shapes, so classification based on shape depends on the accurate description of the more subtle features in a shape.

Histogram techniques are a simple and efficient way of extracting statistical information. This paper discusses two histogram-based shape descriptors, the Edge Co-occurrence Matrix (ECM) and the Contour Co-occurrence Matrix $(\mathrm{CCM})$ and presents experimental results of the application of these descriptors to surface defect classification. Also discussed is the extraction of Haralick features from the feature matrices in order to acquire a shorter feature vector for more efficient computation. A comparison of classification performance is made between these descriptors and other shape descriptors that utilize edge and contour information.

\section{Shape Descriptors}

\subsection{Edge Co-occurrence Matrix}

The Edge Co-occurrence Matrix (ECM) contains second order statistics on edge features in an image. It was introduced in [2, where early results from this work 
were presented. It is similar to the Gray Level Co-occurrence Matrix (GLCM) [3], but instead of a gray level intensity image, it is derived from an edge image, which describes the locations and directions of edges in the original gray-level image. In this respect it is related to the MPEG-7 descriptor Edge Histogram, which contains first order statistics on edge features.

The ECM is calculated as follows. First edge images are formed from the original image by convolving it with the eight Sobel masks [4. The eight masks are needed to retain information on the direction of intensity change in an edge. The eight edge images from the convolutions are combined into one edge image by selecting for each pixel the direction of the strongest edge. This final edge image is then thresholded, so that it contains only the strongest edge pixels. The threshold value is defined as a percentage of the strongest edge value present in the image.

The ECM is then formed from the pairs of edge pixels separated by a given displacement. Let $\mathbf{I}$ be a thresholded edge image and let $\mathbf{d}=\left(d_{x}, d_{y}\right)$ be a displacement vector. Then the Edge Co-occurrence Matrix $\mathbf{H}^{E C M}$ is defined as a matrix, where the $(i, j)$ th element is the number of edge pixels with direction $i$ separated from an edge pixel with direction $j$ by the displacement vector $\mathbf{d}$,

$$
H_{i j}^{E C M}=\#\{\mathbf{x} \mid I(\mathbf{x})=i, I(\mathbf{x}+\mathbf{d})=j\},
$$

where \# is the number of elements in the set and $\mathbf{x}=(x, y)$ runs through the edge image $\mathbf{I}$. Since the edges were detected in 8 directions, the size of the ECM is $8 \times 8$.

Since only the relative distances and directions of pixel pairs are used, the matrix is translation invariant. However, it is not scale or rotation invariant. The sum of the matrix elements is the number of edge pixel pairs found, and even if the matrix is normalized to unit sum, the feature is scale sensitive due to the length of the displacement. However, normalization makes it possible to interpret the matrix as a probability distribution, from which various features can be calculated.

Examples of ECMs calculated from defect images are shown in Figure 1(a).

\subsection{Contour Co-occurrence Matrix}

The Contour Co-occurrence Matrix (CCM), introduced in [5], contains second order statistics on the directionality of the contours of objects in an image. It resembles the ECM and the GLCM, but instead of a two-dimensional image, the co-occurrence information is calculated from the Freeman chain code [6] of the contour of an object. In this regard, it is related to the Chain Code Histogram $(\mathrm{CCH})$ 7] that can be regarded as the CCM's first-order counterpart.

The first step in calculating the CCM of an object is to generate the chain code of its contour. The starting point of the contour is not stored, so the resulting feature descriptor is translation invariant.

The co-occurrence matrix is then formed from the pairs of links separated by a given displacement. Let $A$ be a chain of length $n$ and let $d$ be a displacement, i.e. 


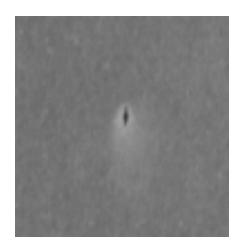

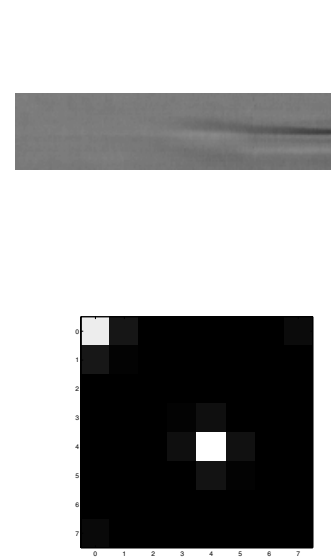

(a) ECM

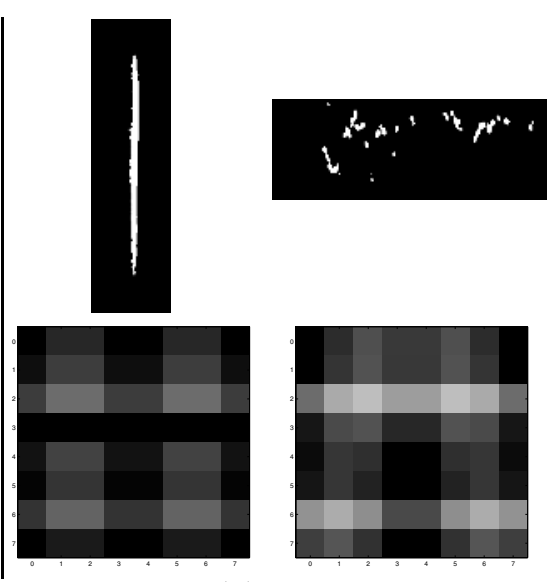

(b) CCM

Fig. 1. Example images of surface defects and the ECMs and CCMs calculated from them

the difference between two chain link indices (not the distance, i.e. the absolute value of the difference). Then the edge co-occurrence matrix $\mathbf{H}^{C C M}$ is defined as a matrix, where the $(i, j)$ th element is the number of instances of a link with value $i$ separated from a link with value $j$ by the displacement $d$,

$$
H_{i j}^{C C M}=\#\left\{k \mid a_{k}=i, a_{k+d(\bmod n)}=j\right\},
$$

where \# is the number of elements in the set and $k$ runs through the values $0,1, \ldots n-1$. Because the chain is derived from a closed contour, the index $k$ and displacement $d$ are summed modulo $n$, so that the displacement wraps around the chain's arbitrary starting point. Since the chain code is octal, the size of the CCM is $8 \times 8$.

Two basic variations of the CCM may be considered, based on whether the displacement $d$ is constant over all examined contours (let this be called the CCM1), or dependent on the length of the chain, i.e. $d=c n$, where $c$ is a real number in the range [0,1[ (let this be called the CCM2). If the sum of the CCM's elements is normalized to unity, the matrix represents the joint probability of the link values $i$ and $j$ occurring at link indexes with the difference $d$. Thus normalized, the CCM2 becomes scale invariant.

Examples of CCMs calculated from defect images are presented in Figureप(b).

\subsection{Other Shape Descriptors}

Other shape descriptors considered in this paper are taken from the MPEG-7 standard, formally named "Multimedia Content Description Interface" [8]. They are well standardized descriptors that are used in searching, identifying, filtering and browsing images or video in various applications. The features are: 
- Edge Histogram (EH) calculates the amount of vertical, horizontal, 45 degree, 135 degree and non-directional edges in 16 sub-images of the picture, resulting in a total of 80 histogram bins.

- Contour-based Shape (CBS) consists of a set of peak coordinates derived from a Curvature Scale Space (CSS) representation of a contour, and the eccentricities and circularities of the contour and its convex prototype, which is created by repeatedly low-pass filtering the contour.

- Region-based Shape (RBS) utilizes a set of 35 Angular Radial Transform (ART) coefficients that are calculated within a disk centered at the center of the image's Y channel.

In addition to the MPEG-7 shape features we also tested three other shape descriptors that we have used previously for defect image classification and retrieval:

- Simple Edge Histogram (SEH) is similar to its MPEG-7 counterpart, but instead of dividing an image into several sub-images, it is calculated like the ECM, for the whole image.

- Simple Shape Descriptor (SSD) 9 consists of several simple descriptors calculated from an object's contour. The descriptors are convexity, principal axis ratio, compactness, circular variance, elliptic variance, and angle.

- Chain Code Histogram (CCH) [7] is an 8-dimensional histogram calculated from the Freeman chain code of a contour. It is the first-order equivalent of the CCM.

\section{$3 \quad$ Experiments}

\subsection{Experimental Setup}

Experiments were carried out with two image databases containing defect images, one from a metal web inspection system and the other from a paper web inspection system. All images are grayscale images, supplied with binary mask images containing segmentation information. The contours of the objects were extracted from the segmentation masks using inner boundary tracing with 8connectivity. The images have different kinds of defects and their sizes vary according to the size of a defect. Classification of defects is based on the cause and type of a defect, and different classes can therefore contain images that are visually dissimilar in many aspects. The paper defect database has 1204 images. They are preclassified into 14 different classes with between 63 and 103 images in all of the classes but one which has only 27 images. The metal defect database has 2004 images. They are preclassified into 14 different classes, with each class containing from 101 up to 165 images. The databases were provided by ABB Oy. More information on these databases can be found e.g. in [10,11].

All tests of the classification performance of the feature were made with K-Nearest Neighbor leave-one-out cross-validation (KNN-LOOCV), using the Euclidean distance. Each feature vector is in turn selected to be classified, while 
the remaining vectors form the training set. The test vector is classified by voting among the $K$ nearest vectors in the training set, using the ground truth information supplied with the images. All tests here use the value $K=5$. The classification success rate in a given class is the percentage of images belonging to the class that have been classified correctly. The average or overall success rate is the mean of the success rates of the classes weighted by the number of images in the classes.

\subsection{Preliminary Experiments}

Some intial tests were made to determine good values for the parameters of the features. These include the displacements for both features and the edge detection threshold for the ECM. A comparison was made between the two versions of the CCM.

The optimum edge detection threshold for these datasets was found to be approximately 15\%, meaning that any detected edge pixels with a strength less than $15 \%$ of the strongest edge present in the image were discarded. This value is highly dependent on the nature of the problem and the image generation process, and is not necessarily the optimum value for any other dataset.

The first comparison is between ECMs using a "full circle" arrangement of eight displacement vectors with a distance of one, an asymmetrical "half circle" version of the same with four vectors, a symmetrical arrangement with vectors $(1,1)$ and $(-1,-1)$, and an asymmetrical version with the vector $(1,1)$. The ECMs were normalized to unit sum. The average classification rates were $59 \%$ for the vector $(1,1), 52 \%$ for the combination $(1,1)$ and $(-1,-1), 59 \%$ for the full circle and $51 \%$ for the half circle.

The most important thing to notice is that the asymmetrical ECMs give significantly better results than the symmetrical ones. Also significant is that reducing the number of displacement vectors from four to one has very little effect on classification performance. This is important since the time required to calculate the ECMs is directly dependent on the number of displacement vectors.

Several different combinations of edge pixel pair displacements were experimented with. Increasing the displacement distance reduced classification success rates, which is to be expected, because the further apart two pixels are, the less likely they are to have any meaningful relationship to each other, and also the less likely it is that they are both edge pixels, since a significant portion of the image contains no edges. It was found that restricting the displacements to only two, vectors $(1,1)$ and $(2,2)$, and concatenating the resulting vectors gave nearly as good results as any other reasonable combination of multiple displacements, with the advantage of being relatively fast to compute and resulting in a relatively short feature vector.

With the CCM the smallest displacements are not likely to give optimal results. In the ECM, the greater the distance, the less likely it is for an edge pixel to be paired with another edge pixel, and the less likely it is for the occurrence of such a pair to represent a significant relationship between those pixels. In the CCM all chain links represent points on the contour of an object, and all link 
pairs have a definite relationship, depending on the displacement between them and the shape and size of the contour. At different distances, different features of the contours are represented in the CCM, and the displacements that give the best results need to be determined individually for each set of data.

Based on experimental results, the displacements 10 and 20 were chosen for the CCM1 in the metal database, and the displacements 20 and 40 in the paper database. The resulting matrices were concatenated to form the feature descriptor. For the CCM2, the relative displacements $0.10,0.20,0.30$, and 0.40 were chosen, and the matrices were summed together to form the feature vector. For more details, see 5 .

Classification results using the descriptors CCM1 and CCM2 as developed above are presented in Table 1. Although the CCM1 performed better, it also required more care in selecting the displacements. If optimizing the selection of displacements is not possible, e.g. the database grows during use, and the initial set is not representative of the actual pool of data being used, then the CCM2 is probably more reliable, due to the use of relative displacements. In this case, using the CCM1 with optimized displacements gives a slight advantage. In the remaining experiments only the CCM1 will be used, and will be referred to simply as the CCM.

Table 1. Comparison between the CCM1 and the CCM2

\begin{tabular}{|l|c|c|c|c|}
\hline & \multicolumn{4}{|c|}{ Classification success rates (\%) } \\
& CCM1 unnorm. & CCM1 norm. & CCM2 unnorm. & CCM2 norm. \\
\hline Metal & 53 & 49 & 51 & 47 \\
\hline Paper & 56 & 58 & 55 & 54 \\
\hline
\end{tabular}

\subsection{Haralick Features}

Using multiple displacement vectors and forming the feature vector by concatenating matrices can result in a long feature vector. Although the performance of the feature can be improved by increasing the information in the feature vector, computations are more efficient with shorter vectors, which may be crucial in real-time applications. One way to make shorter vectors while retaining the information necessary for good performance is to extract from the matrix a set of features which are commonly used with the GLCM. These include angular second moment, contrast, correlation, sum of squares, inverse difference moment, sum average, sum variance, sum entropy, entropy, difference variance, difference entropy, information measures of correlation 1, information measures of correlation 2, and maximal correlation coefficient, which are the original Haralick features [3], and cluster shade and cluster prominence from the additional features introduced by Conners and Harlow [12. While the GLCM is symmetric, the ECM and the CCM are not, which means that one of the original Haralick 
features, sum of squares, needs to be calculated for both axes of the matrix. These features are called simply sum of squares 1 and sum of squares 2 . In order to calculate these features, the matrix must be normalized so that it can be interpreted as a probability distribution.

A matrix obviously cannot be described completely with this set of features, but performance comparable to that of the original matrix can be achieved with a suitable selection from the features. The extracted feature set may even have a better classification success rate than the original matrix, but since the objective is to reduce the feature vector length, it is more interesting to see what is the smallest set that still performs at an acceptable level compared with the original matrix.

The set of all 17 features was used as a starting point, and a greedy algorithm was used to eliminate features from the set. On each iteration, the classification was performed with all such subsets of the current feature set where one feature had been removed. The subset that gave the highest overall success rate was used as the set for the next iteration. Since the values of the features have very different ranges, the feature vector was whitened using PCA, resulting in a vector where each component has zero mean and unit variance. Figure 2 shows graphs for the classification rate at each iteration.

The lowest number of features sufficient for achieving the same approximate level of performance as the original matrix was four, with the ECM in the paper database. In the metal database the same number of features performed at the level of the normalized matrix, which is only a little weaker than the unnormalized matrix, so four was selected as the size of the Haralick feature sets. The sets are listed in Table 2,

With the CCM, the performance of the feature set is not as good in comparison to the original matrix as it is with the ECM. This is especially evident in the metal database, where the performance level of the unnormalized matrix was not reached at any point, and the level of the normalized matrix required nine features.
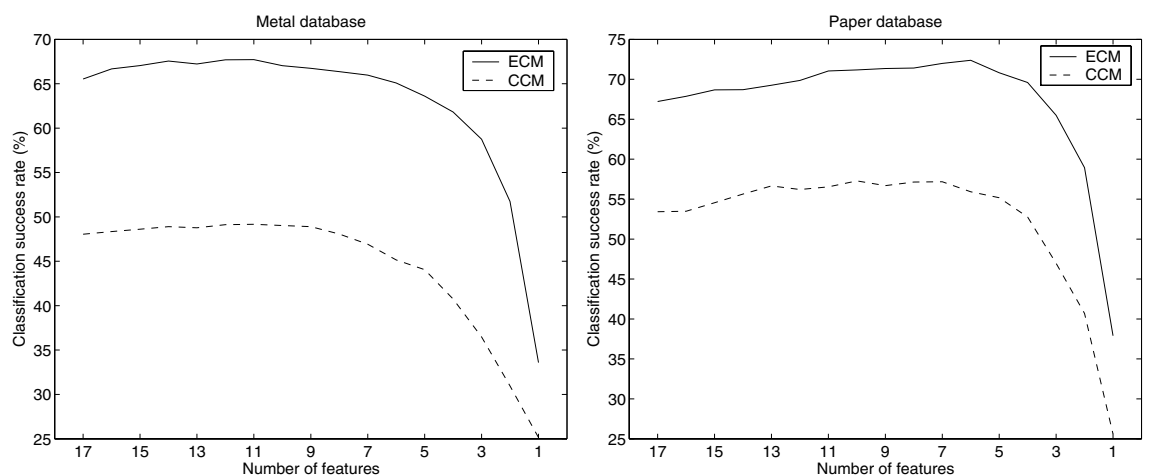

Fig. 2. Classification success rates as a function of the number of features during the feature elimination 
Table 2. The sets of features used in classification

\begin{tabular}{|c|c|c|}
\hline & Metal & Paper \\
\hline $\mathrm{ECM}$ & $\begin{array}{l}\text { Sum of squares } 1 \\
\text { Sum of squares } 2 \\
\text { Information measures of correlation } 2 \\
\text { Sum average }\end{array}$ & $\begin{array}{l}\text { Sum of squares } 1 \\
\text { Sum of squares } 2 \\
\text { Information measures of correlation } 1 \\
\text { Sum entropy }\end{array}$ \\
\hline$\overline{\mathrm{CCM}}$ & $\begin{array}{l}\text { Difference entropy } \\
\text { Information measures of correlation } 1 \\
\text { Cluster shade } \\
\text { Cluster prominence }\end{array}$ & $\begin{array}{l}\text { Inverse difference moment } \\
\text { Entropy } \\
\text { Information measures of correlation } 2 \\
\text { Cluster prominence }\end{array}$ \\
\hline
\end{tabular}

\subsection{Feature Comparisons}

The ECM and CCM were compared with other shape descriptors with similar approaches. The unnormalized version of the ECM, the normalized version, and the Haralick features calculated from the normalized ECM were compared with the Edge Histogram (EH) and the Simple Edge Histogram (SEH). The unnormalized CCM, the normalized version and the extracted Haralick features were compared with the Contour-based Shape (CBS), the Region-based Shape (RBS), the Chain Code Histogram (CCH), and the Simple Shape Descriptor (SSD). The results are shown in Tables 3 and 4 .

The results show the superiority of the ECM over the EH. In the metal database, the unnormalized ECM scores 18 percentage units higher than the $\mathrm{EH}$. In the paper database, the difference is 9 percentage units. However, when compared with the $\mathrm{SEH}$, the differences are 23 percentage units for metal and 29 percentage units for paper, so the difference between the first order SEH and second order ECM appears consistent between the databases. The considerable advantage the $\mathrm{EH}$ has over the $\mathrm{SEH}$ in the paper database may be related to the fact that paper defects have more clearly defined edges than metal defects. The stronger contrast between edge regions and non-edge regions could result in less correlation between the feature vector components and increased discriminatory power for the $\mathrm{EH}$ in the paper database.

The unnormalized ECM is better than the normalized ECM in both databases, and in part this may be caused by its greater sensitivity to scale. However, in some classes the normalization decreases the success rate significantly, but extracting Haralick features from the normalized matrix increases the success rate again, even exceeding the success rate of the unnormalized ECM. This suggests that the decrease in success rate associated with normalization is not simply a result of a loss of information.

In the CCM comparisons the best results were obtained with the CCM, and the second best with the SSD. In the metal database the unnormalized CCM scored 4 percentage units higher than the normalized CCM, and 11 percentage units higher than the SSD. However, in the paper database the normalized CCM 
Table 3. KNN classification results in the metal and paper databases

\begin{tabular}{|l|c|c|c|c|c|}
\hline & \multicolumn{4}{|c|}{ Classification success rates (\%) } \\
& ECM & ECM & ECM & EH & SEH \\
unnorm & norm. & Haralick & & \\
\hline Metal & 67 & 63 & 62 & 49 & 44 \\
\hline Paper & 69 & 64 & 70 & 60 & 40 \\
\hline
\end{tabular}

Table 4. KNN classification results in the metal and paper databases

\begin{tabular}{|c|c|c|c|c|c|c|c|}
\hline & \multicolumn{7}{|c|}{ Classification success rates (\%) } \\
\hline & $\begin{array}{c}\text { CCM } \\
\text { unnorm }\end{array}$ & $\begin{array}{l}\mathrm{CCM} \\
\text { norm. }\end{array}$ & $\left|\begin{array}{c}\text { CCM } \\
\text { Haralick }\end{array}\right|$ & SSD & $\mathrm{CCH}$ & CBS & RBS \\
\hline Metal & 53 & 49 & 42 & 42 & 36 & 31 & 20 \\
\hline Paper & 56 & 58 & 52 & 52 & 40 & 49 & 46 \\
\hline
\end{tabular}

scored 2 percentage units higher than the unnormalized CCM and only 6 percentage units higher than the SSD. The lowest scorers are the CCH, the CBS and the RBS. Their rankings are inconsistent between the databases, the RBS being the worst in the metal database and the $\mathrm{CCH}$ being the worst in the paper database. The advantage of the CCM over the first order $\mathrm{CCH}$ is consistent between the databases, 17 percentage units for metal and 18 percentage units for paper.

\section{Conclusions}

Statistical shape features can be used to describe irregular objects such as surface defects. The Edge Co-occurrence Matrix (ECM) and Contour Co-occurrence Matrix (CCM) perform better in surface defect classification tasks than their first order counterparts and other shape descriptors, including the the Region-based Shape (RBS) and Contour-based Shape (CBS) descriptors from the MPEG-7 standard. The ECM performs better than the CCM, which may be explained by the fact that the defect images do not have well-defined boundaries.

The length of the feature vectors can be decreased by extracting Haralick features from the co-occurence matrices. No set that would be conveniently small and would also give good results for each descriptor-database-combination could be found. The effort of computing the features and finding a good set should be considered if Haralick features are used.

Acknowledgments. The financial supports of the Technology Development Centre of Finland (TEKES's grant 40102/04) and our industrial partner ABB Oy (J. Rauhamaa) are gratefully acknowledged. 


\section{References}

1. Marshall, S.: Review of shape coding techniques. Image and Vision Computing 7 (1989) 281-294

2. Rautkorpi, R., Iivarinen, J.: A novel shape feature for image classification and retrieval. In: Proceedings of the International Conference on Image Analysis and Recognition, Porto, Portugal (2004)

3. Haralick, R., Shanmugam, K., Dinstein, I.: Textural features for image classification. IEEE Transactions on Systems, Man, and Cybernetics SMC-3 (1973) 610-621

4. Sonka, M., Hlavac, V., Boyle, R.: Image Processing, Analysis and Machine Vision. Chapman \& Hall Computing, London (1993)

5. Rautkorpi, R.: Shape features in the classification and retrieval of surface defect images. Master's thesis, Helsinki University of Technology (2005)

6. Freeman, H.: Computer processing of line-drawing images. Computing Surveys 6 (1974) $57-97$

7. Iivarinen, J., Visa, A.: Shape recognition of irregular objects. In Casasent, D.P., ed.: Intelligent Robots and Computer Vision XV: Algorithms, Techniques, Active Vision, and Materials Handling. Proc. SPIE 2904 (1996) 25-32

8. Manjunath, B.S., Salembier, P., Sikora, T., eds.: Introduction to MPEG-7: Multimedia Content Description Interface. John Wiley \& Sons Ltd. (2002)

9. Iivarinen, J., Visa, A.: An adaptive texture and shape based defect classification. In: Proceedings of the 14th International Conference on Pattern Recognition. Volume I., Brisbane, Australia (1998) 117-122

10. Pakkanen, J., Ilvesmki, A., Iivarinen, J.: Defect image classification and retrieval with MPEG-7 descriptors. In Bigun, J., Gustavsson, T., eds.: Proceedings of the 13th Scandinavian Conference on Image Analysis. LNCS 2749, Gteborg, Sweden, Springer-Verlag (2003) 349-355

11. Iivarinen, J., Rautkorpi, R., Pakkanen, J., Rauhamaa, J.: Content-based retrieval of surface defect images with PicSOM. International Journal of Fuzzy Systems 6 (2004) 160-167

12. Conners, R., Harlow, C.: Toward a structural textural analyser based on statistical methods. Computer Graphics and Image Processing 12 (1980) 224-256 